\title{
Resultado visual comparativo entre dois aparelhos de excimer laser: Summit Apex Plus e Aesculap-Meditec Mel 70 em cirurgia de miopia e astigmatismo
}

\author{
Summit Apex Plus versus Aesculap-Meditec Mel 70 in the treatment of \\ Myopia and astigmatism
}

\author{
Marcia Domingues Fernandes ${ }^{1}$ \\ Marta Sartori ${ }^{2}$ \\ Mauro Campos ${ }^{3}$
}

\footnotetext{
${ }^{1}$ Mestranda e Médica do Setor de Cirurgia Refrativa da Universidade Federal de São Paulo (UNIFESP).

2 Doutora em Oftalmologia e Médica do Setor de Cirurgia Refrativa da Universidade Federal de São Paulo (UNIFESP).

${ }^{3}$ Professor Livre-docente e Chefe do Setor de Cirurgia Refrativa da Universidade Federal de São Paulo (UNIFESP).

Endereço para correspondência: Marcia D. Fernandes Rua Apinagés, 731, apto. 41 - São Paulo (SP)

CEP 05017-000 - E-mail: fdmarcia.ops@terra.com.br

Recebido para publicação em 02.09.2002

Aceito para publicação em 07.04.2003
}

\begin{tabular}{|c|}
\hline RESUMO \\
\hline 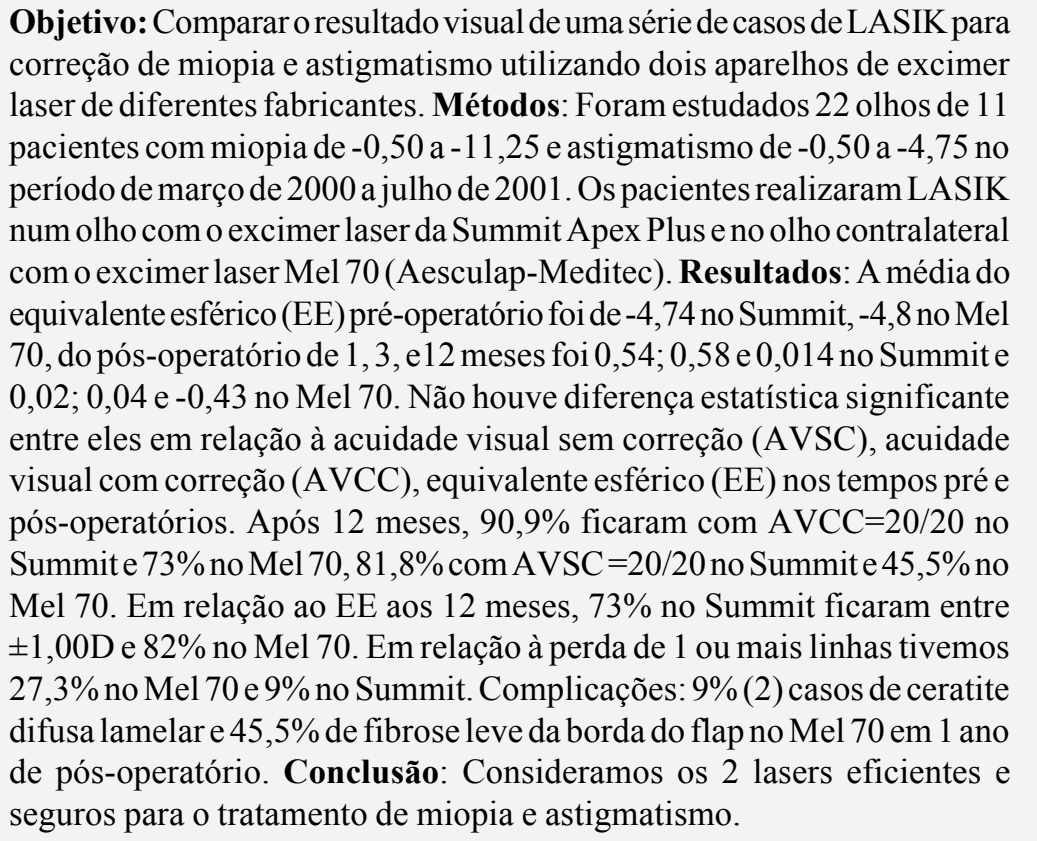 \\
\hline
\end{tabular}

Descritores: Miopia/cirurgia; Astigmatismo/cirurgia; Estudo comparativo; Keratomileusis in situ assistida por excimer laser

\section{INTRODUÇ̃̃̃O}

Durante a última década avanços em cirurgia refrativa a laser têm envolvido principalmente a correção de miopia e astigmatismo.

A primeira geração dos excimers lasers de fluoreto de argônio engloba os aparelhos que utilizam feixe largo ou "broad beam", diafragma, zonas ópticas pequenas, máscaras para correção de astigmatismo e correção esfero-cilíndrica simples como é o caso do laser Summit Apex Plus. A segunda geração engloba os lasers de "scanning" ou "small spot", que utilizam zonas ópticas maiores e não utilizam máscaras para a correção de astigmatismo que é o caso do Meditec Aesculap Mel 70(1).

Escolhemos como técnica cirúrgica o LASIK (laser in situ keratomileusis), pois é o mais comumente utilizado em cirurgia refrativa e tem se mostrado ao longo dos anos efetivo para correção de miopia e astigmatismo de leve a moderado. Esta técnica cirúrgica tem se refinado concomitantemente à evolução dos lasers. 
O objetivo deste estudo é comparar o resultado visual em uma série consecutiva de casos de LASIK (laser in situ keratomileusis), utilizando dois aparelhos de excimer laser de diferentes fabricantes, um de primeira e outro de segunda geração.

\section{MÉTODOS}

Foram estudados prospectivamente 11 olhos de 11 pacientes com miopia variando de $-0,50$ a $-11,25$ e astigmatismo variando de $-0,50$ a $-4,25 \mathrm{D}$ tratados com laser de fluoreto de argônio 193 nm da Summit Apex Plus (Summit Technology, In, Wasltham, MA) e 11 olhos de 11 pacientes com miopia variando de $-0,50$ a $-8,50$ e astigmatismo variando de $-0,50$ a $-4,75$ tratados no Mel 70 (Aesculap-Meditec, Jana, Germany) no período de março de 2000 a julho de 2001.

Os pacientes foram submetidos à avaliação pré-operatória que incluía: registro da acuidade visual com e sem correção, refração estática, biomicroscopia, tonometria de aplanação, mapeamento de retina, videoceratoscopia computadorizada e paquimetria ultra-sônica. Os usuários de lentes de contato gelatinosas e rígidas foram orientados a descontinuá-las 7 e 14 dias antes dos exames pré-operatórios.

Os critérios de inclusão foram idade acima de 18 anos, ausência de patologias oculares ou sistêmicas que afetem a cicatrização corneana e refração estável por pelo menos 1 ano.

A técnica cirúrgica utilizada para todos os olhos foi laser in situ keratomileusis (LASIK). Os microcerátomos utilizados foram: 1) motorizado horizontal da Chiron (Chiron Corneal Shaper) e o vertical da Moria (Carriazo-Barraquer) manual e elétrico. Foi calculado um leito residual de pelo menos $250 \mu \mathrm{m}$.

O seguimento pós-operatório foi de 1, 3 e 12 meses.

A medicação pós-operatória foi: colírio de dexametasona $0,1 \%$ associado a tobramicina $4 \mathrm{x}$ dia por 15 dias.

Foram avaliados no pós-operatório: registro da acuidade visual sem correção e corrigida, refração estática, biomicroscopia, tonometria e ceratoscopia computadorizada.

\section{MÉTODO ESTATÍSTICO}

Para compararmos possíveis diferenças entre os tempos para as variáveis previamente definidas usamos o teste não paramétrico para K amostras não independentes de Friedman, complementado, quando necessário, pelo Teste de Comparações Múltiplas.

Quando comparamos possíveis diferenças entre os dois grupos em cada tempo usamos o teste não paramétrico para amostras independentes de Mann-Wittney.

O nível de rejeição para a hipótese de nulidade foi fixado sempre em um valor $\leq 0,05(5 \%)$.

As médias foram calculadas e apresentadas a título de informação. Não se calculou desvio padrão, pois, usando-se testes não paramétricos estamos pressupondo que as variáveis em causa não se comportam como curva de Gauss e portanto não há sentido o seu cálculo.
Para compararmos possíveis diferenças entre os 2 grupos quanto ao nível de visão e perda de linha usamos o teste do Quiquadrado $\left(\mathrm{X}^{2}\right)$ para tabelas de contingência e quando havia restrições de Cochran as mesmas foram transformadas em tabelas de associação, ou então, o Teste de Fisher.

\section{RESULTADOS}

A idade dos pacientes variou de 22 a 49 anos (média de 32 anos), sendo 7 do sexo feminino e 4 do masculino. Foram estudados 22 olhos de 11 pacientes, sendo 11 olhos tratados no Summit e 11 olhos no Mel 70. A refração pré-operatória e pós-operatória é mostrada na tabela 1 .

Foi realizado o teste de Mann-Wittney para a comparação dos 2 grupos pré-operatórios para sabermos se as amostras eram semelhantes, e não houve diferença significante entre eles em relação a $\operatorname{AVSC}(p=1,000), \operatorname{AVCC}(p=0,329)$ e EE $(\mathrm{p}=0,692)$.

A média do equivalente esférico(EE) pré-operatório foi de $-4,74$ no Summit, -4,8 no Mel 70, do pós-operatório de 1,3 e 12 meses foi 0,$54 ; 0,58$ e 0,014 no Summit e 0,$02 ; 0,04$ e $-0,43$ no Mel 70. Comparando os 2 grupos não houve diferença significante $(\mathrm{p}=0,500)$ entre eles (Gráfico 1$)$.

\section{Grupo do Laser Summit: (Teste de Friedmann)}

1. Equivalente esférico (EE): houve diferença significante entre os valores pré-operatórios e os pós-operatórios $(\mathrm{p}=0,000)$, e não houve diferença entre os tempos pós-operatórios de 1,3 e 12 meses.

2. Acuidade Visual sem Correção (AVSC): também houve diferença significante somente entre o pré e o pós $(\mathrm{p}=0,000)$.

3. Acuidade Visual com Correção (AVCC): não houve diferença significante entre o pré e o pós $(\mathrm{p}=0,486)$, nem entre os tempos pós-operatórios.

\section{Grupo do Laser Mel 70 (semelhante ao anterior):}

1. EE: houve diferença significante somente entre o pré e o pós $(\mathrm{p}=0,000)$.

2. AVSC: houve diferença significante somente entre o pré e o pós $(\mathrm{p}=0,000)$

3. AVCC: não houve diferença significante entre o pré e o pós $(\mathrm{p}=0,036)$ e os demais tempos pós-operatórios.

\section{Comparação entre SUMMIT x MEL 70 (Teste de Mann-Wittney):}

1. EE: não houve diferença significante entre os tempos pós-operatórios de 1 mês $(\mathrm{p}=0,169), 3$ meses $(\mathrm{p}=0,193)$ e 12 meses $(\mathrm{p}=0,679)$.

2. AVSC: não houve diferença significante com 1 mês $(\mathrm{p}=0,529), 3$ meses $(\mathrm{p}=0,833)$ e 12 meses $(\mathrm{p}=0,573)$.

3. AVCC: não houve diferença significante com 1 mês $(p=0,542), 3$ meses $(p=0,466)$ e 12 meses $(p=0,252)$. 


\begin{tabular}{|c|c|c|c|c|c|c|}
\hline \multicolumn{7}{|c|}{ Tabela 1} \\
\hline \multirow[b]{2}{*}{ Pacientes } & \multirow[b]{2}{*}{ Olhos } & \multirow[b]{2}{*}{ Grupos Laser } & \multirow[b]{2}{*}{ RX pré (EE) } & \multicolumn{3}{|c|}{ Pós-operatório em meses (EE) } \\
\hline & & & & 1 & 3 & 12 \\
\hline \multirow[t]{2}{*}{1} & OD & Summit & $-4,50$ & $-1,25$ & $-1,25$ & $-1,50$ \\
\hline & OE & Mel 70 & $-4,50$ & 0,50 & 0 & $-0,60$ \\
\hline \multirow[t]{2}{*}{2} & OD & Summit & $-4,00$ & $-0,25$ & 1,00 & 1,25 \\
\hline & OE & Mel 70 & $-4,25$ & $-1,00$ & 0 & 0,25 \\
\hline \multirow[t]{2}{*}{3} & OE & Summit & $-3,75$ & 0,75 & 0 & 0,90 \\
\hline & OD & Mel 70 & $-3,75$ & $-0,30$ & $-0,40$ & $-0,10$ \\
\hline \multirow[t]{2}{*}{4} & OD & Summit & $-4,00$ & 1,00 & 0,50 & $-0,25$ \\
\hline & OE & Mel 70 & $-4,00$ & 1,25 & 1,00 & 0,25 \\
\hline \multirow[t]{2}{*}{5} & OD & Summit & $-3,50$ & 0,50 & 0,75 & $-0,10$ \\
\hline & OE & Mel 70 & $-3,50$ & 0,50 & 1,00 & 0,50 \\
\hline \multirow[t]{2}{*}{6} & OD & Summit & $-4,30$ & 1,90 & 1,70 & 0,75 \\
\hline & OE & Mel 70 & $-4,50$ & 0,50 & 0,25 & 0 \\
\hline \multirow[t]{2}{*}{7} & OE & Summit & $-4,50$ & 0,25 & 1,00 & 0,25 \\
\hline & OD & Mel 70 & $-6,10$ & $-2,30$ & $-0,50$ & $-1,70$ \\
\hline \multirow[t]{2}{*}{8} & OD & Summit & $-4,30$ & 1,00 & 1,00 & 0 \\
\hline & $\mathrm{OE}$ & Mel 70 & $-6,10$ & 0,70 & $-0,10$ & 0 \\
\hline \multirow[t]{2}{*}{9} & OD & Summit & $-4,50$ & 1,00 & 1,25 & $-0,25$ \\
\hline & OE & Mel 70 & $-4,20$ & $-1,00$ & $-0,80$ & $-2,50$ \\
\hline \multirow[t]{2}{*}{10} & OE & Summit & $-11,25$ & 0,25 & $-0,25$ & $-1,30$ \\
\hline & OD & Mel 70 & $-8,90$ & 0,25 & $-0,75$ & $-1,00$ \\
\hline \multirow[t]{2}{*}{11} & $\mathrm{OE}$ & Summit & $-2,60$ & 0,75 & 0,75 & 0,40 \\
\hline & OD & Mel 70 & $-3,50$ & 1,10 & 0,70 & 0,25 \\
\hline
\end{tabular}

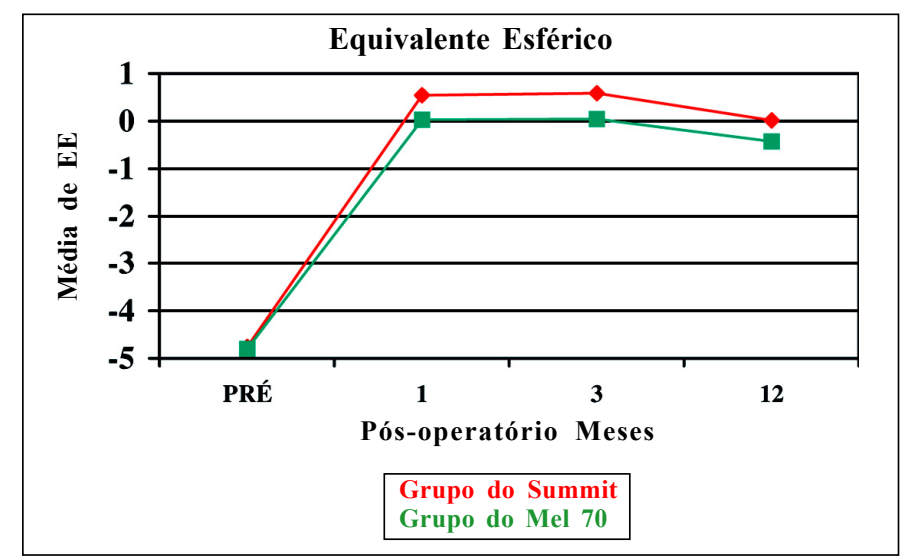

Gráfico 1

Comparando os 2 grupos quanto ao nível de AVCC pelo Teste de Fisher, com 1 ano de pós-operatório, não houve diferença significante $(p=0,500)$ se AVCC $\geq 20 / 40(p=0,500)$ ou AVCC $=20 / 20(p=0,500)$.

Comparando os 2 grupos quanto a AVSC pelo Teste de Fisher com 1 ano de pós-operatório não houve diferença significante se AVSC $\geq 20 / 40(p=0,500)$ e se AVSC $=20 / 20(p=0,091)$.

Quanto à porcentagem de olhos de acordo com o nível de acuidade visual, vide tabela 2 .

\section{Equivalente esférico após 1 ano de pós-operatório:}

A maioria ficou entre $\pm 1,00 \mathrm{D}(73 \%$ no Summit e $82 \%$ no Mel 70) (Tabela 3).

\section{Perda de linha de visão com correção após 1 ano:}

Comparando os 2 grupos pelo Teste de Fisher não houve diferença significante entre eles $(\mathrm{p}=0,250)$, apesar de a perda maior ocorrer no grupo do Mel 70 (Tabela 4).

\section{Complicações}

Cinco olhos $(45,5 \%)$ no grupo do Mel 70 apresentaram epitelização da borda do flap no pós-operatório recente que resultou em fibrose periférica tênue após 1 ano; 2 olhos $(18,2 \%)$ no grupo do Mel 70 tiveram ceratite lamelar difusa (DLK) no pós-operatório imediato, sendo que em 1 paciente teve que ser realizada lavagem da interface do flap no $3^{\circ}$ dia de pós-operatório com AVSC final de 20/25, e a outra paciente foi tratada clinicamente com AVSC final de 20/20.

\section{DISCUSSÃO}

Nosso estudo demonstra grande precisão para a correção de miopia e astigmatismo nos 2 lasers onde a média refracional residual após 1 ano foi 0,014 no Summit e -0,43 no Mel 70, comparável a outros estudos: como $^{(2)}$ que estudando miopias leves e moderadas obtiveram EE residual -0,29; $\mathrm{e}^{(3)} \mathrm{em}$ baixas miopias obtiveram - 0,11 e médias miopias $-0,42$.

Quanto à acuidade visual corrigida (AVCC) com 1 ano de cirurgia $90,9 \%$ dos olhos operados no Summit e $73 \%$ dos olhos no Mel 70 ficaram com AVCC $=20 / 20$, se considerarmos AVCC $\geq 20 / 40: 90,9 \%$ dos olhos no Summit contra $100 \%$ no Mel 70. Já em relação a acuidade visual sem correção 


\begin{tabular}{|c|c|c|c|c|c|c|c|c|}
\hline \multicolumn{9}{|c|}{ Tabela 2} \\
\hline \multirow{2}{*}{$\begin{array}{l}\text { Meses pós- } \\
\text { operatório }\end{array}$} & \multirow[t]{2}{*}{ Grupos } & \multirow[t]{2}{*}{ N Olhos } & \multicolumn{3}{|c|}{ AVSC } & \multicolumn{3}{|c|}{ AVCC } \\
\hline & & & $=20 / 20$ & $20 / 40$ a $20 / 25$ & $<20 / 40$ & $=20 / 20$ & $20 / 40$ a $20 / 25$ & $<20 / 40$ \\
\hline \multirow[t]{2}{*}{1} & Summit & 11 & $5(45,5 \%)$ & $4(36,4 \%)$ & $2(18,2 \%)$ & $10(90,9 \%)$ & 0 & $1(9 \%)$ \\
\hline & Mel 70 & 11 & $3(27,3 \%)$ & $7(63,6 \%)$ & $1(9,0 \%)$ & $7(63,6 \%)$ & $4(36,4 \%)$ & 0 \\
\hline \multirow[t]{2}{*}{3} & Summit & 11 & $7(63,6 \%)$ & $2(18,2 \%)$ & $2(18,2 \%)$ & $9(81,8 \%)$ & $1(9,0 \%)$ & $1(9 \%)$ \\
\hline & Mel 70 & 11 & $5(45,5 \%)$ & $6(54,5 \%)$ & 0 & $6(54,5 \%)$ & $5(45,4 \%)$ & 0 \\
\hline \multirow[t]{2}{*}{12} & Summit & 11 & $9(81,8 \%)$ & 0 & $2(18,2 \%)$ & $10(90,9 \%)$ & 0 & $1(9 \%)$ \\
\hline & Mel 70 & 11 & $5(45,5 \%)$ & $5(45,5 \%)$ & $1(09,0 \%)$ & $8(72,7 \%)$ & $3(27,3 \%)$ & 0 \\
\hline
\end{tabular}

\begin{tabular}{|c|c|c|c|c|c|c|}
\hline \multicolumn{7}{|c|}{ Tabela 3} \\
\hline $\begin{array}{l}\text { Meses } \\
\text { pós-operatório }\end{array}$ & Grupos & $\mathbf{N}$ olhos & $\pm 0,5 \mathrm{D}$ & $\pm 1,00 \mathrm{D}$ & $\pm 2,00 \mathrm{D}$ & $\pm 3,00$ \\
\hline \multirow[t]{2}{*}{1} & Summit & 11 & $4(36,4 \%)$ & $5(82 \%)$ & $2(100 \%)$ & 0 \\
\hline & Mel 70 & 11 & $5(45,4 \%)$ & $4(82 \%)$ & $1(91 \%)$ & $1(100 \%)$ \\
\hline \multirow[t]{2}{*}{3} & Summit & 11 & $4(36,4 \%)$ & $4(73 \%)$ & $3(100 \%)$ & 0 \\
\hline & Mel 70 & 11 & $5(45,5 \%)$ & $4(82 \%)$ & $1(91 \%)$ & $1(100 \%)$ \\
\hline \multirow[t]{2}{*}{12} & Summit & 11 & $6(54,5 \%)$ & $2(73 \%)$ & $3(100 \%)$ & 0 \\
\hline & Mel 70 & 11 & $7(63,6 \%)$ & $2(82 \%)$ & $1(91 \%)$ & $1(100 \%)$ \\
\hline
\end{tabular}

\begin{tabular}{|lcc|}
\hline & Tabela 4 & \\
12 meses pós-operatório & Summit & Mel 70 \\
Perda de 1 linha AVCC & 0 & $3(27,3 \%)$ \\
Perda de 2 linhas AVCC & $1(9 \%)$ & 0 \\
Total olhos & $1(9 \%)$ & $3(27,3 \%)$ \\
\hline
\end{tabular}

(AVSC): $81,8 \%$ no Summit contra $45,5 \%$ no MEL 70 ficaram com AVSC $=20 / 20$, apesar de parecer melhor não foi significante ( $\mathrm{p}=0,091)$; mas em todos os tempos de pós-operatórios o grupo do Summit apresentou na AVSC e na AVCC maior porcentagem de olhos com melhor visão em relação ao Mel 70 (Tabela 2). Outros autores tiveram: $88,2 \% \operatorname{AVSC} \geq 20 / 20^{(2)}$; AVSC $\geq 20 / 2044,6 \%$ em Lasik simultâneo e 36,6\% em Lasik sequencial e $89 \%$ de AVSC $\geq 20 / 40$ em Lasik sequencial e 91,8\% de AVSC $\geq 20 / 40$ em Lasik simultâneo ${ }^{(4)}$.

Em contra-partida, em relação ao equivalente esférico (EE) a maior porcentagem de olhos entre $\pm 0,5 \mathrm{D}$ em todos os tempos de pós-operatórios está no grupo do Mel 70 (Tabela 3). Talvez a explicação para o grupo do Mel 70 ter a pior AVSC mas com menor EE seja uma maior irregularidade corneana no pósoperatório, no entanto faltaria uma avaliação do estudo topográfico que infelizmente não foi realizada. No estudo $\mathrm{de}^{(4)}$ $53,1 \%$ dos olhos está entre $\pm 0,50 \mathrm{D}$ em Lasik sequencial, $58,6 \%$ em Lasik simultâneo e entre $\pm 1,00 \mathrm{D}, 82,6 \%$ em Lasik sequencial e 84,5\% em Lasik simultâneo.

Em relação à perda de linhas de visão com correção, somente 1 olho ( $9 \%$ ) perdeu duas linhas no aparelho da Summit; pois era o único paciente alto míope com $A V C C=20 / 30$ no préoperatório e passou a ter $\mathrm{AVCC}=20 / 60$ após 1 ano de cirurgia. $\mathrm{O}$ outro olho deste paciente também apresentou perda de linha no Mel 70. No trabalho de ${ }^{(3)}$ não foi encontrada perda de linha de visão no grupo dos altos míopes, somente na baixa miopia $3,7 \%$ e $12,4 \%$ na média miopia ${ }^{(4)}$; encontraram $4 \%$ no Lasik com perda de $\geq 2$ linhas de visão ${ }^{(5)}$ mostraram uma maior perda de linha no grupo de altos míopes. Nosso índice de perda de linha foi maior no grupo do $\operatorname{Mel} 70(27,3 \%)$.

Tivemos complicações pós-operatórias somente no grupo do laser Mel 70 como descrevemos anteriormente, que foram os 2 casos ( $9 \%$ considerando os 22 olhos operados) de "diffuse lamellar keratitis" (DLK), resolvidas com sucesso. Esta complicação tem sido descrita com uma freqüência de $1,8 \%$ a $4 \%$ dos $\operatorname{casos}^{(6-8)}$.

Tivemos 5 olhos (45,5\% dos olhos no Mel 70 e $22,7 \%$ se considerarmos os 22 olhos operados) com fibrose periférica do flap, provavelmente, pelo aumento da área de tratamento deste tipo de laser sem o devido aumento do diâmetro do flap com os microcerátomos utilizados. Felizmente não houve progressão da epitelização inicial, sendo observada a partir do 3 mês de pós-operatório uma regressão progressiva ficando somente uma linha tênue na borda do flap. Um autor ${ }^{(9)}$ refere que o crescimento epitelial tem sido descrito em $2 \%$ a $15 \%$ depois de Lasik para miopias e $0,3 \%$ a 31,4\% depois de Lasik para hipermetropias. Segundo ${ }^{(10)}$ a incidência varia de $0,3 \%$ a $15 \%$, chegando a $31 \%$ no retratamento e descreve que o crescimento epitelial após cirurgia refrativa é uma complicação bem conhecida, podendo ser devido a inúmeras causas entre elas a abrasão epitelial intra-operatória (que acreditamos ser o caso dos pacientes deste estudo pelo aumento da área tratada pelo laser Mel 70 ser maior do que o corte do flap), e que os vacúolos de células epiteliais isoladas dentro da interface do flap têm um limitado potencial de proliferação e tende a se resolver espontaneamente dentro de meses sem perda de visão ou necessidade de intervenção cirúrgica. 


\section{CONCLUSÃO}

Basicamente não houve diferença estatística significante entre os 2 tipos de laser em relação a acuidade visual com e sem correção, equivalente esférico e perda de linha de visão. Acreditamos que tanto o laser da Summit quanto o laser Mel 70 são eficientes e seguros para o tratamento de miopia e astigmatismo leve e moderado. Infelizmente temos um número muito pequeno de casos, sendo realmente difícil encontrar pacientes operados com lasers de marcas diferentes com seguimento longo, para compararmos os resultados; além da dificuldade técnica e ética para utilização de diferentes lasers num mesmo paciente.

\section{ABSTRACT}

Purpose: To compare the visual result in consecutive case series between two different excimer laser equipments in LASIK for myopia and astigmatism. Methods: We analyzed the results of LASIK of 11 eyes of 11 patients with myopia ranging from -0.50 to -11.25 and astigmatism from -0.50 to -4.25 who were submitted to Summit Apex Plus and 11 eyes of 11 patients with myopia ranging from -0.50 to -8.75 and astigmatism from -0.50 to -4.75 submitted to Meditec-Aesculap Mel 70.The follow-up was 1, 3 and 12 months. Results: Preoperatively the mean spherical equivalent was -4.75 for Summit and -4.8 for Mel 70. At 1, 3 and 12 months after surgery, the residual error for the Summit group was $0.54 ; 0.58$ and 0.014 and for the Mel 70 group it was $0.02 ; 0.04$ and -0.43 . There were no statistically significant differences between two excimer laser equipments regarding: spherical equivalent (SE), uncorrected visual acuity (UCVA), best-corrected visual acuity (BCVA) pre- and postoperatively. One year after surgery $90.9 \%$ presented BCVA $=20 / 20$ for Summit and 73\% for Mel 70, $81.8 \%$ had UCVA $=20 / 20$ for Summit and $45.5 \%$ for Mel 70 . The final mean postoperative SE was $73 \%$ within $\pm 1.00 \mathrm{D}$ for Summit and $82 \%$ within $\pm 1,00 \mathrm{D}$ for Mel 70 . Loss of one or two lines BCVA was $9 \%$ for Summit and $27.3 \%$ for Mel 70. Complications: $9 \%$ cases of diffuse lamellar keratitis and $45.5 \%$ of epithelial ingrowth for Mel 70. Discussion: Both Summit Apex Plus and Meditec-Aesculap Mel 70 were found to be similar, safe and efficient for the correction of myopia and astigmatism.

Keywords: Myopia/surgery; Astigmatism/surgery; Keratomileusis, laser in situ; Comparative study

\section{REFERÊNCIAS}

1. McDonald MB, Carr JD, Frantz JM, Kozarsky AM, Maguen E, Nesburn AB, et al. Laser in situ keratomileusis for myopia up to -11 diopters with up to -5 diopters of astigmatism with the summit autonomous LADARVision excimer laser system. Ophthalmology 2001;108:309-15.

2. Forseto AS, Nosé RAM, Nosé W. PRK versus LASIK para correção de miopia baixa e moderada. Arq Bras Oftalmol 2000;63:257-62.

3. Kwitko S, Marinho D, Raskin R, Sprinz S, Rabin M, Rymer S, et al. Lasik para correção de miopia, astigmatismo e hipermetropia. Arq Bras Oftalmol 2000;63:9-17.

4. Waring GO, Carr JD, Stulting RD, Thompson KP, Wiley W. Prospective randomized comparison of simultaneous and sequential bilateral laser in situ keratomileusis for the correction of myopia. Ophthalmology 1999;106:732-45.

5. Knorz MC, Wiesinger B, Leirmann A, Sliberth V, Liesenholf H. Laser in situ keratomileusis for moderate and high myopia and myopic astigmatism. Ophthalmology 1998;105:932-40.

6. Weldon W, Haw MD, Manche EE. Late onset diffuse lamellar keratitis associated with an epithelial defect in six eyes. J Refract Surg 2000;16:744-8.

7. Lin RT, Maloney RK. Flap complications associated with lamellar refractive surgery [commented on Am J Ophthalmol 1999;127:202-4]. Am J Ophthalmolo 1999;127:129-36.

8. Maloney RK, Binder OS, Machat JJ, et al. Sterile interface inflammation after laser in situ keratomileusis: experience and opinions. J Refract Surg 1998;14: 661-6.

9. Vesaluoma MH, Petroll M, Pérez-Santonja JJ, Valle TU, Alió JL, Tervo TM. Laser in situ keratomileusis flap margin: wound healing and complications imaged by in vivo confocal microscopy. Am J Ophthalmology 2000; 130:564-73

10. Haw WW, Manche EE. Treatment of progressive or recurrent epithelial ingrowth with ethanol following laser in situ keratomileusis. J Refract Surg 2001;17:63-8.

\title{
ABO ELETRÔNICO
}

\author{
Novo site
}

\section{Acesso: http://www.abonet.com.br}

